\title{
Condition Domain
}

National Cancer Institute

\section{Source}

National Cancer Institute. Condition Domain. NCI Thesaurus. Code C154631.

A condition represents a patient's diagnosed and self-reported health conditions and diseases. The patient's medical history and current state may both be represented. 